\title{
PIETSCH INTEGRAL OPERATORS DEFINED ON INJECTIVE TENSOR PRODUCTS OF SPACES AND APPLICATIONS
}

\author{
by DUMITRU POPA
}

(Received 30 January, 1996)

\begin{abstract}
For $X$ and $Y$ Banach spaces, let $X \otimes_{\epsilon} Y$, be the injective tensor product. If $Z$ is also a Banach space and $U \in L\left(X \otimes_{\epsilon} Y, Z\right)$ we consider the operator

$$
U^{*}: X \rightarrow L(Y, Z), \quad\left(U^{*} x\right)(y)=U(x \otimes y), x \in X, y \in Y .
$$

We prove that if $U \in P I\left(X \otimes_{\epsilon} Y, Z\right)$, then $U^{*} \in I(X, P I(Y, Z))$. This result is then applied in the case of operators defined on the space of all $X$-valued continuous functions on the compact Hausdorff space $T$. We obtain also an affirmative answer to a problem of J. Diestel and J. J. Uhl about the RNP property for the space of all nuclear operators; namely if $X^{*}$ and $Y$ have the $R N P$ and $Y$ can be complemented in its bidual, then $N(X, Y)$ has the $R N P$.
\end{abstract}

An operator $U \in L(X, Y)$ is called a Pietsch integral operator if there exists a $Y$-valued vector measure with bounded variation on the Borel subsets of $\left(U_{X^{*}}\right.$, weak*) such that: $U(x)=\int_{U_{x^{*}}} x^{*}(x) d G\left(x^{*}\right)$ for each $x \in X$ and the Pietsch integral norm of $U$ is: $\|U\|_{p i n t}=\inf |G|\left(U_{X^{*}}\right)$. It is well known that the class of all Pietsch integral operators with the Pietsch integral norm is a normed ideal of operators in the sense of A. Pietsch, which in the sequel will be denoted by $\left(P I,\|\|_{p i n t}\right)$. Also $U \in P I(X, Y)$ if and only if for each $\epsilon>0, U$ admits a factorisation of the form

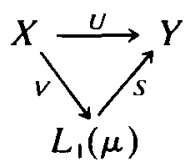

where $V \in I\left(X, L_{1}(\mu)\right), S \in L\left(L_{1}(\mu), Y\right)$ and $\|V\|_{\text {int }} \leq\|U\|_{\text {pint }}+\epsilon,\|S\| \leq 1$; see [2] for details.

For the definition of integral operator, absolutely summing operator, nuclear operator and their basic properties see [2] or [4]. By $I(),,\|\|_{i n t}$, (resp. $\left(A s,\|\|_{a s}\right)$, $\left(N,\|\|_{n u c}\right)$ we denote the normed ideal of all integral operators (resp. absolutely summing operators, nuclear operators). For all notations and notions used and not defined we refer the reader to [2]. Given $U \in L\left(X \otimes_{e} Y, Z\right)$ we consider the operator $U^{\sharp}: X \rightarrow L(Y, Z)$ defined by $\left(U^{*} x\right)(y)=U(x \otimes y), x \in X, y \in Y$, that is evidently linear and continuous. Also for a given normed ideal of operators $\Im$ and $U \in \Im\left(X \otimes_{\epsilon} Y, Z\right)$ we have $U^{\sharp} x \in \Im(Y, Z)$, for any $x \in X$. Indeed, if $x \in X$, let $V_{x} \in L\left(Y, X \otimes_{\epsilon} Y\right)$ be the operator $V_{x}(y)=x \otimes y, y \in Y$. Since $U^{\sharp} x=U V_{x}$, by the ideal property of $\Im$ we obtain $U^{\sharp} x \in \Im(Y, Z)$. Hence for a normed ideal of operators $\Im$ and $U \in L\left(X \otimes_{\varepsilon} Y, Z\right)$ we can consider the assertions

(a) $U \in \Im\left(X \otimes_{\epsilon} Y, Z\right)$,

(b) $U^{\#} \in \Im(X, \Im(Y, Z))$.

In the sequel for the normed ideal of Pietsch integral operators we study the connection between (a) and (b); see also [3], [6], [7] for corresponding work on other normed ideals. 
THEOREM 1. If $U \in P I\left(X \otimes_{\epsilon} Y, Z\right)$, then $U^{\#} \in I(X, P I(Y, Z))$ and $\|U\|_{\text {int }} \leq\|U\|_{\text {pinr }}$

Proof. We make first a remark. If $W$ can be complemented in its bidual by a norm one projection, then $I\left(X \otimes_{\epsilon} Y, W\right)=I(X, I(Y, W))$, which follows easily using Theorem 2.1 from [3]. Now if $U \in P I\left(X \otimes_{\epsilon} Y, Z\right)$, then for each fixed $\epsilon>0, U$ admits a factorisation

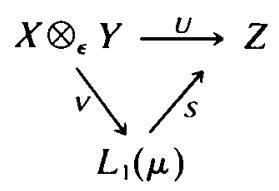

where $V \in I\left(X \otimes_{\epsilon} Y, L_{1}(\mu)\right), S \in L\left(L_{1}(\mu), Z\right)$ and $\|V\|_{\text {int }} \leq\|U\|_{\text {pint }}+\epsilon,\|S\| \leq 1$. (Here $\mu$ is a regular Borel measure on some compact Hausdorff space $\Omega$.). See [2, Theorem 11, p. 168]. Using the above remark for $W=L_{1}(\mu)$ we obtain that $V^{\#} \in I\left(X, I\left(Y, L_{1}(\mu)\right)\right)$ and $\left\|V^{\#}\right\|_{\text {int }}=\|V\|_{\text {int }}$. However Grothendieck's theorem shows that $I\left(., L_{1}(\mu)\right)=P I\left(., L_{1}(\mu)\right)$ and \|\|$_{\text {int }}=\|\|_{\text {pint }}$, (See [2, Theorem p. 558].) Thus we have the factorisation

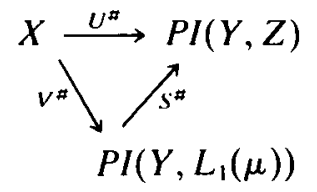

where $S^{\#}(A)=S A, A \in P I\left(Y, L_{1}(\mu)\right)$ and, by the ideal property of the class of all integral operators, we obtain $U^{\#} \in I(X, P I(Y, Z))$ and $\left\|U^{\#}\right\|_{\text {int }} \leq\left\|V^{\#}\right\|_{\text {int }}\left\|S^{\#}\right\| \leq\left\|V^{\#}\right\|_{\text {int }}\|S\| \leq$ $\|V\|_{\text {int* }}$

Thus $\left\|U^{\#}\right\|_{\text {int }} \leq\|U\|_{\text {pint }}+\epsilon$, hence $\left\|U^{\#}\right\|_{\text {int }} \leq\|U\|_{\text {pinr }}$

In the sequel, by $T$ we denote a compact Hausdorff space and $C(T, X)$ will be the Banach space of all $X$-valued continuous functions on $T$ under the supremum norm. For $X=\mathbf{R}$ (or $\mathbf{C}$ ) we note that $C(T, X)=C(T)$. By $\Sigma$ we denote the $\boldsymbol{\sigma}$-field of all Borel subsets of $T$. It is well known [2, p. 182] that any $U \in L(C(T, X), Y)$ has a representing finitely additive vector measure $G: \Sigma \rightarrow L\left(X, Y^{* *}\right)$. For $U \in L(C(T, X), Y)$, we consider the operator

$$
U^{\sharp}: C(T) \rightarrow L(X, Y), \quad\left(U^{\sharp} \varphi\right)(x)=U(\varphi x), \quad \varphi \in C(T), \quad x \in X .
$$

Since $C(T, X)=C(T) \otimes_{\epsilon} X$, from Theorem 1 we obtain the following corollary.

Corollary 2. Let $U \in L(C(T, X), Y)), U^{\#}$ be as above and $G$ be the representing measure of $U$. We consider the following assertions:

(a) $U \in P I(C(T, X), Y)$;

(b) $U^{\sharp} \varphi \in P I(X, Y)$ for each $\varphi \in C(T)$ and $U^{\sharp} \in P I(C(T), P I(X, Y))$;

(c) $G(E) \in P I(X, Y)$ for each $E \in \Sigma$ and $G: \Sigma \rightarrow P I(X, Y)$ has bounded variation with respect to the Pietsch integral norm on $P I(X, Y)$.

Then we have $(a) \Rightarrow(b) \Rightarrow(c)$ and, in this case the following inequality holds: $\left\|U^{\#}\right\|_{\text {pint }}=|G|_{\text {pint }}(T) \leq\|U\|_{\text {pint }}$.

Proof. For the implication (a) $\Rightarrow(\mathrm{b})$ we use Theorem 1 and the well known facts $P I(C(T),)=.A s(C(T),$.$) and \|\|_{p i n t}=\|\|_{a s}$. See Theorem 12 of [2, p. 169]. For the 
implication (b) $\Rightarrow$ (c) we again use Theorem 12 of $[2$, p. 69] and the obvious fact that the representing measure of $U$ in the hypothesis of (b) coincides with that of $U$. The relations: $\left\|U^{*}\right\|_{\text {pint }}=|G|_{\text {pint }}(T) \leq\|U\|_{\text {pint }}$ are also true. In this way arises the following conjecture.

Conjecture 3. If $U \in L(C(T, X), Y)$ has the representing measure $G$ which satisfies the conditions

(1) $G(E) \in P I(X, Y)$ for each $E \in \Sigma$ and

(2) $G: \Sigma \rightarrow P I(X, Y)$ has bounded variation with respect to the Pietsch integral norm,

then it follows that $U \in P I(C(T, X), Y)$.

If $Y$ can be complemented in its bidual, then it is well known that we have $I(., Y)=P I(., Y)$ Corollary 10 of $[2$, p. 235] and hence using the result of P. Saab from [6] we obtain that this with supplementary hypothesis about $Y$ Conjecture 3 is true. In the sequel we describe the Question 5 from the paper of P. Saab [6] as the Saab conjecture.

Saab conjecture. If $Y$ has the $R N P$ and $U \in L(C(T, X), Y)$ has the representing measure $G$ which satisfies the conditions

(1) $G(E) \in N(X, Y)$ for each $E \in \Sigma$ and

(2) $G: \Sigma \rightarrow N(X, Y)$ has bounded variation with respect to the nuclear norm, it follows that $U \in N(C(T, X), Y)$.

Recall also the following open problem of Diestel and Uhl. See [2, p. 258].

Diestel-Uhl conjecture. If $X^{*}$ and $Y$ have the RNP, then the space of all nuclear operators from $X$ to $Y$ also has the RNP.

The following theorem establishes a connection between these problems.

THEOREM 4. Conjecture 3 is true implies Saab conjecture is true implies Diestel-Uhl conjecture is true.

Proof. Conjecture 3 is true implies Saab conjecture is true; it is obvious since, if $Y$ has the $R N P$, then $P I(., Y)=N(., Y)$ and \|\|$_{\text {pint }}=\|\|_{\text {nuc }}$. See Theorem 2 of $[2, \mathrm{p} .175]$.)

Saab conjecture is true implies Diestel-Uhl conjecture is true. Let $X$ and $Y$ be Banach spaces such that $X^{*}$ and $Y$ have the $R N P$. Let $\Sigma$ be the Borel subsets of $[0,1]$ and $G \in \operatorname{rcabv}\left(\Sigma, N(X, Y),\|\|_{n u c}\right)$. Let $U: C([0,1], X) \rightarrow Y$ be the operator $U(f)=\int_{0}^{1} f d G$, $f \in C(T, X)$. Then $U$ is a linear and continuous operator and $G$ is its representing measure.

Since $Y$ has the $R N P$ and the Saab conjecture is true then, $U$ will be a nuclear operator. Since $X^{*}$ has the $R N P$ from [5, Theorem 1] or [7, Theorem 6] we obtain that $G: \Sigma \rightarrow\left(N(X, Y),\|\|_{n u c}\right)$ has a Bochner integrable derivative $g \in L_{1}\left(\mu, N(X, Y),\|\|_{n u c}\right)$, where $\mu=|G|_{\text {nuc }}$. Thus $N(X, Y)$ has the $R N P$.

In [2, Theorem 5 p. 249] and [1, Theorem 7 p. 119] are given positive answers to the Diestel-Uhl conjecture, with supplementary hypotheses about $X$ or $Y$. Since as we have seen the Conjecture 3 is true when $Y$ can be complemented in its bidual from Theorem 4 we obtain the following corollary which is another positive answer to the Diestel-Uhl conjecture different from those given in [1] and [2].

Corollary 5. If $X$ and $Y$ are Banach spaces such that $X^{*}$ and $Y$ have the RNP and $Y$ can be complemented in its bidual, then $N(X, Y)$ also has the $R N P$. 


\section{REFERENCE'S}

1. K. T. Andrews, The Radon Nikodym property for spaces of nuclear operators, J. London Math. Soc. 28 (1983), 113-122.

2. J. Diestel and J. J. Uhl, Vector measures, Math Surveys No 15, (A.M.S., 1977).

3. S. Montgomery-Smith and Paulette Saab, $p$-summing operators on injective tensor products of spaces, Proc. Royal Soc. Edinburgh Sect. 120 (1992), 283-296.

4. A. Pietsch, Operator ideals (Veb. Deutscher Verlag der Wiss., Berlin, 1978).

5. D. Popa, Nuclear operators in $C(T, X)$, Studii si Cercet. Mat. 42(1) (1990), 47-50.

6. P. Saab, Integral operators on spaces of continuous vector valued functions, Proc. Amer. Math. Soc. 111 (1991) 1003-1013.

7. P. Saab and B. Smith, Nuclear operators on spaces of continuous vector valued functions, Glasgow Math., 33 (1991), 223-230.

8. Charles Swartz, Absolutely summing and dominated operators on spaces of vector-valued continuous functions, Trans. Amer. Math. Soc. 179 (1973), 123-131.

Department of Mathematics

UNIVERSITY OF CONSTANTA

8700 CONSTANTA

Romania 